\title{
Analyse fréquentielle et nouvelle cartographie des maxima annuels de pluies journalières au Bénin
}

\author{
Alain I. AGUE ${ }^{1 *}$ et Abel AFOUDA ${ }^{1,2}$ \\ ${ }^{I}$ Chaire Internationale en Physique Mathématique et Applications (CIPMA-Chaire UNESCO), \\ Université d'Abomey-Calavi, 072 BP : 50 Cotonou, Bénin. \\ ${ }^{2}$ Laboratoire d'Hydrologie Appliquée (LHA), Université d'Abomey-Calavi, Bénin. \\ "Auteur correspondant, E-mail : ibikalain@yahoo.fr; Tel :00 22966512088
}

\section{RESUME}

Les pluies extrêmes sont des phénomènes rares qui sont la cause des inondations ayant des impacts sur la population. La présente étude vise à estimer et cartographier les maxima annuels des pluies journalières au Bénin. A cet effet, les données de 35 stations ont été utilisées et couvrent globalement la période de 1921 à 2001. Cinq lois statistiques ont été utilisées pour l'analyse fréquentielle. Le choix du meilleur modèle a été fait sur la base de la comparaison numérique. Les résultats ont montré une prédominance des lois Gumbel et Lognormale. Dans l'ensemble, on note que les lois de probabilité des maxima annuels de pluies journalières, n'obéissent pas spécifiquement à un régime pluviométrique donné. On constate également que l'évolution des quantiles de pluie est proportionnelle à celles des périodes de retour car en effet, les périodes de retour augmentent avec les quantiles de pluie. Les valeurs les plus élevées sont généralement observées au niveau du Littoral, au Centre Est et au Nord Ouest du Bénin. Ainsi, la présente étude permet de disposer d'une nouvelle cartographie des maxima annuels des pluies journalières pour les périodes de retour 5, 10, 50 et 100 ans au Bénin.

(C) 2015 International Formulae Group. All rights reserved.

Mots clés : Pluies extrêmes, analyse fréquentielle, Bénin.

\section{INTRODUCTION}

Etat de l'Afrique de l'Ouest, la république du Bénin est située dans la zone tropicale entre l'équateur et le tropique du Cancer, entre les latitudes $6^{\circ} 30^{\prime}$ et $12^{\circ} 30^{\prime}$ Nord et les longitudes $1^{\circ}$ et $3^{\circ} 40^{\prime}$ Est. Elle est caractérisée par trois zones climatiques (Figure 1): une zone subéquatoriale dans le sud (entre les parallèles $6^{\circ} 30^{\prime}$ et $7^{\circ} \mathrm{N}$ ) de régime climatique bimodal à quatre (04) saisons (deux saisons de pluies et deux sèches); une zone soudano-guinéenne de transition, localisée au centre du pays entre les parallèles $7^{\circ}$ et $10^{\circ} \mathrm{N}$ à régime climatique à cheval sur les régimes uni-modal et bimodal ; une zone soudanienne au-delà de la latitude $10^{\circ} \mathrm{N}$ caractérisée par un régime climatique uni-modal à deux (02) saisons (une sèche et une pluvieuse).

A l'instar de nombreux pays du monde en général et de l'Afrique en particulier, le Bénin connaît les affres du changement climatique dont la maîtrise et l'évaluation des risques passe en partie par la connaissance des pluies extrêmes comme les hauteurs maximales annuelles des pluies journalières. 
L'analyse de ce paramètre climatique se fait à partir de plusieurs approches dont l'approche statistique qui est souvent utilisée. C'est le cas des différentes études sur les pluies extrêmes menées à travers le monde par Panigrahi et Sudhindra (2002) en Inde, Koutsoyiannis (2000, 2004a, 2004b, 2005) en Grèce, Bouvier et al. (2005) en France. En Afrique, on peut citer les études réalisées par Benkhaled (2007) en Algérie, Van Vyver et Démarrée (2010) au Congo Kinshasa et Goula et al. (2010) en Côte d'Ivoire.

Dans son rapport de synthèse sur les pluies journalières de fréquence rare, le CIEH (1985) a produit les cartes des isohyètes $P_{10}$ et $\mathrm{P}_{100}$ des pays membres qui sont encore en vigueur pour le dimensionnement des ouvrages hydrauliques.

Au Bénin, l'étude réalisée par le CIEH (1985), souvent utilisée pour le dimensionnement des ouvrages hydrauliques, concernent des périodes largement dépassées de nos jours. De plus, l'étude statistique des valeurs extrêmes a toujours été menée de façon conventionnelle à l'aide de la loi de Gumbel. Cette situation ne garantie pas une sécurité des aménagements. La présente étude a pour objectif de :

- Faire l'analyse fréquentielle des maxima annuels de pluies journalières au Bénin ;

- Elaborer la carte de validité des lois statistiques;

- Produire la carte des isohyètes des pluies extrêmes pouvant être utilisée pour la planification des ouvrages hydrauliques et pour les projets de développement durable au Bénin.

\section{MATERIEL ET METHODES}

\section{Données de pluies journalières}

Les données utilisées sont celle de 35 stations (pluviométriques et pluviographiques), toutes localisées au Bénin (Figure1) provenant de la base de données météorologiques du Bénin reçue à l'ASECNA. Ces stations ont été retenues à cause du nombre d'années d'observations égales au moins à 50 ans et de la qualité des données pluviométriques définie par un taux de lacune inférieure à $10 \%$. Elles couvrent globalement la période allant de 1921 à 2001 et les séries de maxima annuels de pluies journalières sont constituées.

\section{Méthodologie de l'analyse fréquentielle}

L'analyse fréquentielle passe en premier lieu par l'appréciation de la qualité des séries à posséder une fonction de distribution en utilisant les tests de stationnarité de Kendall (Aka et al., 1996), d'indépendance de Wald-Wolfowitz (Hache et al., 1999) et d'homogénéité de Wilcoxon (Siegel, 1956).

Plusieurs lois peuvent être utilisées comme fonction de distribution des valeurs extrêmes. Plusieurs études dans le monde comme en Afrique tropicale ont montré la prédominance de la loi de Gumbel. Dans la présente étude, nous nous sommes appuyés sur les lois statistiques GEV, Gumbel, Lognormale, Pearson type III et Log-Pearson type III. Le Tableau 1 présente les fonctions de densité de probabilité de ces lois.

La méthode du maximum de vraisemblance a été choisie pour l'estimation des paramètres pour toutes les lois à l'exception de la loi Log-Pearson type III qui a nécessité l'usage de L-Moment.

Vue la complexité que peut présenter la comparaison graphique, nous avons choisi le meilleur modèle sur la base de la comparaison numérique avec la probabilité à postériori et les critères Bayésien (Schwarz, 1978 ; Akaike, 1974) qui se présentent comme suit :

\section{Critère d'information Bayésien (BIC)}

L'expression de ce critère se note : $\mathrm{BIC}=-2 \log (\mathrm{L})+2 \mathrm{k} \log (\mathrm{N})$

Où:

$\mathrm{L}$ : la vraisemblance,

$\mathrm{K}$ : le nombre de paramètres,

$\mathrm{N}$ : la taille de l'échantillon

Il se place dans un contexte bayésien de sélection du modèle probabiliste (Lebartier et Mary-Huard, 2004, cité par Soro et al., 2008) et recherche le modèle $\mathrm{M}_{\mathrm{BIC}}$ qui maximise la 
distribution a posteriori des modèles c'est-àdire le modèle le plus vraisemblable au vu des données.

\section{Critère d'information d'Akaike (AIC)}

L'expression de ce critère se note :

$$
\mathrm{AIC}=-2 \log (\mathrm{L})+2 \mathrm{~K}
$$

Avec :

$\mathrm{L}$ : la vraisemblance,

$\mathrm{K}$ : nombre de paramètres

Il revient à rechercher le modèle faisant le meilleur compromis biais-variance pour le nombre de données $\mathrm{N}$ dont on dispose (Lebartier et Mary-Huard, 2004 cité par Soro et al., 2008).

Les isohyètes ont été tracées par la méthode du krigeage. Elle utilise les relations spatiales existant entre l'ensemble des stations en tenant compte de l'imprécision d'estimation des différentes valeurs. C'est cette méthode qui a été employée dans des études anciennes comme celle de Darricau (1980) au Burkina-Faso et aussi dans des études récentes comme celle de Goula et al., (2007). Afin d'apporter le maximum d'informations pour le tracé des courbes isovaleurs, nous avons pris en compte toutes les stations ayant servi à l'étude.

\section{RESULTATS ET DISCUSSION Test d'hypothèses}

Le test de Kendall a montré qu'il n'y a aucune tendance dans les observations au seuil de défaillance de 5\% sauf à Allada, Bantè, Cotonou ville et Nikki où il est passé à $1 \%$. Par contre, à Savè, il a montré qu'il y a une tendance dans les observations au seuil de 1\%. L'application du test de Wald Wolfowitz indique que les observations sont indépendantes au seuil de $1 \%$ à la station de Sakété, au seuil de 5\% au niveau de toutes les autres stations à l'exception des stations de Boukounbé, Savè et Cotonou-Ville où le test révèle qu'il pourrait avoir de liens entre les observations successives. L'application du test de Wilcoxon-Mann Whitney indique que la moyenne des deux sous échantillons est égale, au seuil de défaillance de $1 \%$ à la station de Savè et $5 \%$ au niveau des 34 autres stations.
On en conclut que les données sont homogènes. Les Résultats au niveau des stations ne sont pas liés à leur appartenance à une zone climatique. Cette observation est identique à celle obtenue en Côte d'Ivoire par Goula et al. (2007).

\section{Evolution de la fonction de probabilité}

Les Figures 2 a, 2 b et 2 c, présentent la probabilité empirique de quelques séries des maxima annuels de pluies, pris dans les trois zones climatiques. L'analyse de la Figure 3 montre qu'il serait difficile de choisir graphiquement la meilleure loi de probabilité, d'où l'utilisation des critères de comparaison AIC et BIC. Cette observation est identique à celle obtenue par Goula et al., (2010). La meilleure loi est celle qui présente les plus faibles valeurs de ces deux critères sur la série de données analysées. Le Tableau 2 présente les critères de comparaison AIC et BIC des cinq lois de probabilité utilisées sur la série des données de la station de Cotonou Aéroport. L'analyse de ce tableau montre que la loi Lognormale est la meilleure loi adaptée à la série des données de la station de Cotonou Ville.

\section{Zone de validité des lois de probabilité}

La Figure 4 présente la loi de probabilité retenue par station avec leur zone de validité. L'analyse de cette carte montre que les distributions de probabilité des pluies journalières maximales annuelles n'obéissent pas spécifiquement à un régime pluviométrique donné. Dans chaque zone climatique, on note au moins trois types de lois de probabilité ayant chacune au moins une zone de validité. Ce résultat concorde avec celui obtenu par Soro et al. (2008) qui a observé que trois lois statistiques (Lognormale, GEV et Gumbel) peuvent être utilisées pour décrire les intensités maximales annuelles de la région d'Abidjan.

De façon générale, on note néanmoins une dominance des lois Gumbel et Lognormale représentées dans les trois zones climatiques. Les GEV, Peason-Type III et Log-Peason type III, quoique faiblement 
représentées, admettent néanmoins des zones de validité sur l'étendue du territoire. Ainsi, la loi GEV s'applique dans une frange au Nord Ouest et au Centre Est du Bénin, qui sont des régions montagneuses respectivement sous le régime Soudanien tropical et Soudanoguinéen. La loi Peason-Type III est représentée de façon lâche, au Nord sous le régime Soudanien et au Sud entre les Latitudes $6,5^{\circ}$ et $7,5^{\circ}$. La loi Log Peason-Type III, quoique très faiblement représentée est néanmoins validée dans la zone de couverture de la station de Tchaourou. On peut donc retenir que l'usage d'une ou au plus deux lois, comme dans les anciennes études, pourrait ne pas permettre, une bonne estimation des quantiles des pluies journalières au Bénin. Cette conclusion concorde avec celle de Goula et al. (2007) qui ont montré que seule la loi de Gumbel ne peut pas être exclusivement utilisée sur l'ensemble du territoire de la Côte d'Ivoire.

\section{Estimation et comparaison des quantiles}

Le Tableau 2 présente les quantiles calculés à partir des distributions des maxima annuels de pluies journalières de 35 stations pluviométriques. Les périodes de retour retenues sont celles de 100 ans, 50 ans, 10 ans et 5 ans. L'on constate que l'évolution des quantiles de pluie est proportionnelle à celles des périodes de retour, car les périodes de retour augmentent avec les quantiles de pluie. Ces résultats sont similaires à ceux obtenus par Soro (2008). Les valeurs de quantiles les plus élevées sont généralement obtenues au niveau des stations côtières puis à Savè et Tanguiéta.

\section{Cartographie des quantiles estimés}

La Figure 5 montre la cartographie des quantiles de pluies journalières extrêmes pour les périodes de retour de 100 ans, 50 ans, 10 ans et 5 ans. L'analyse de ces cartes montre que les valeurs les plus élevées sont surtout sur le littoral entre les latitudes $6^{\circ}$ et $6^{\circ} 30$, au centre Est autour de la latitude $8^{\circ}$ et en plus au Nord Ouest entre les latitudes $10^{\circ}$ et $11^{\circ} 30$ pour les cartes des pluies journalières des périodes de retour 100 et 50 ans. On observe dans ces zones une très forte variabilité des pluies journalières extrêmes caractérisés par le rapprochement des isohyètes. On note en effet, que la distance entre les isohyètes augmente au fur et à mesure que la période de retour diminue. Les observations similaires sont faites par Soro (2010) lors de ses travaux de thèse sur les pluies extrêmes en Côte d'Ivoire. Alors, dans la zone côtière, au regard des différents isohyètes, on peut dire d'une part que la proximité de l'Océan Atlantique a une influence sur les quantiles. Cette zone est le lieu d'une forte pluviométrie à cause de la mousson ouest africaine. Cela est confirmé par les maxima de pluies extrêmes enregistrées aux stations côtières. De plus, les valeurs théoriques (quantiles calculées) à ces différentes stations sont très élevées. Les résultats obtenus au nord Ouest qui est une zone montagneuse caractérisée par la chaine de l'Atakora, rappellent le rôle important joué par l'orographie dans les fortes pluies. Zahar et Laborde (2007), Goula et al. (2007) ont aussi montré l'influence orographique sur les pluies extrêmes. La situation du centre Est pourrait être non seulement due à une influence des collines de la région mais aussi à une intense évapotranspiration engendrée par la densité du couvert végétal et qui est le siège d'une pluviométrie abondante. Ainsi, Les pluies maximales allant de 170 à $260 \mathrm{~mm}$ sont susceptibles de tomber au centre Est du Bénin une fois au moins tous les 100 ans. Au sud du Bénin, jusqu'à la latitude de $7^{\circ}$, on pourrait enregistrer des pluies exceptionnelles de hauteur allant de 115 à $165 \mathrm{~mm}$ au moins une fois tous les 10 ans. Aussi, il importe de noter, en dépit du fait que la période de retour de 50 ans ne soit pas très souvent utilisée dans le dimensionnement des ouvrages hydrauliques, que les pluies maximales allant de 135 à $185 \mathrm{~mm}$ sont susceptibles de tomber au sud, centre Est et au Nord-Ouest du Bénin une fois au moins tous les 50 ans. 


\section{Comparaison des isohyètes actuelles et du CIEH (1985)}

Les isohyètes des pluies journalières centennales et décennales élaborées dans la présente étude ont été comparées à celles du CIEH (1985) généralement utilisées pour le dimensionnement des ouvrages hydrauliques (Figure 6). L'analyse de la Figure 6 montre une différence notable entre les isohyètes proposées par le CIEH et celles de la présente étude. En effet, les isohyètes proposées par le CIEH ne couvrent pas tout le Bénin et sont très espacées comparativement à celles de la présente étude. Goula et al. (2010) ont également noté les mêmes différences entre les isohyètes anciennement proposées par le CIEH en Côte d'Ivoire. Les cartes du CIEH ne montrent pas la situation réelle des pluies journalières centennales comme décennales au Bénin en général et de façon spécifique dans les zones du centre Est et du nord Ouest. Au niveau des pluies centennales, les isohyètes de valeurs élevées et surtout au-delà de 200, ne sont pas représentées sur les cartes du CIEH alors qu'elles sont nettement présentes sur les résultats de la présente étude. Le même scénario est répété au niveau des pluies journalières décennales, surtout au centre Est du Bénin, où nous avons eu les isohyètes 125 et 135 qui sont absentes au niveau de la carte du CIEH. Ces résultats montrent la caducité des résultats du CIEH à efficacement contribuer au dimensionnement des ouvrages hydraulique surtout dans le concept actuelle de changement climatique.

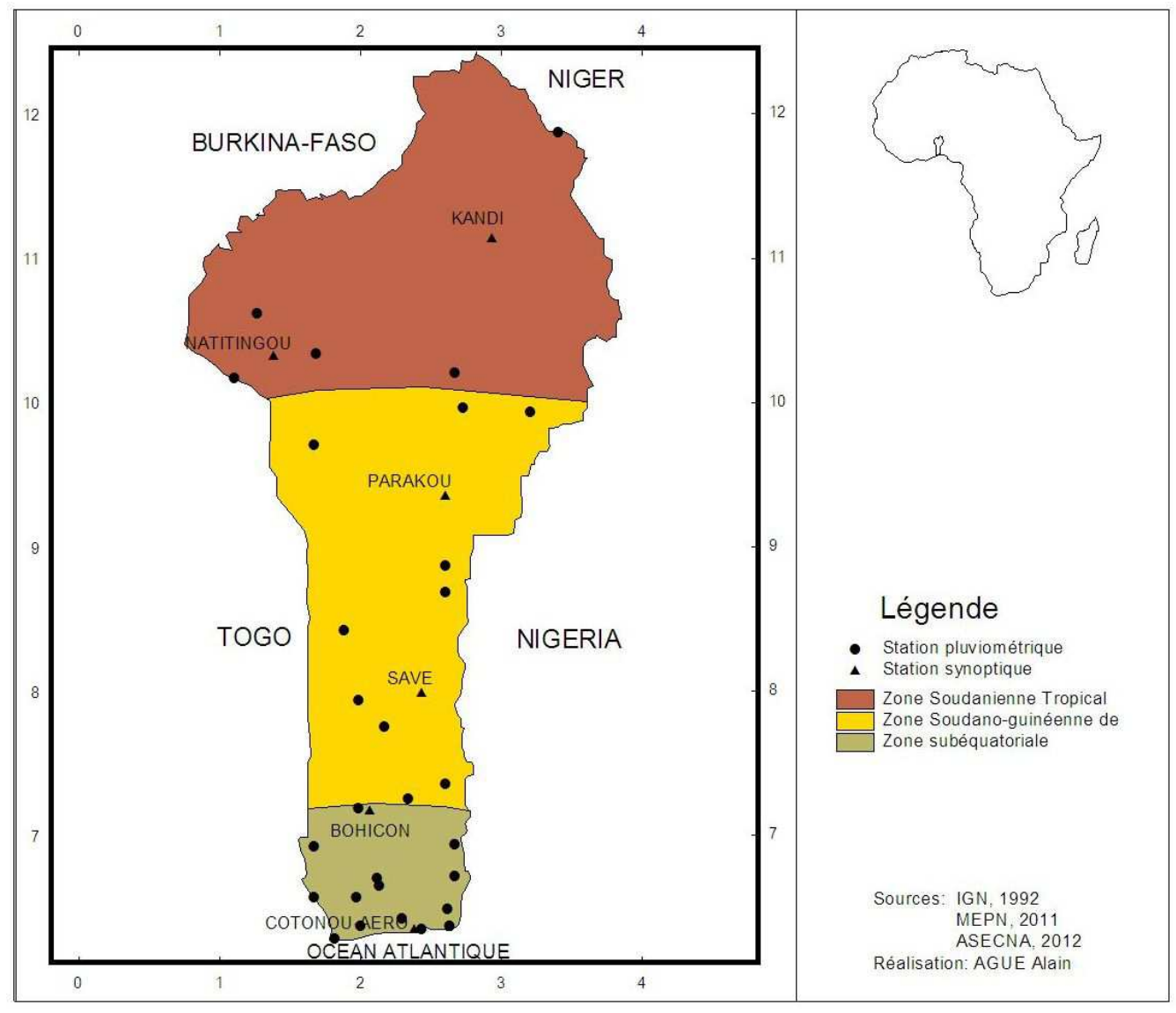

Figure 1 : Localisation des stations d'étude et zonage climatique du Bénin. 


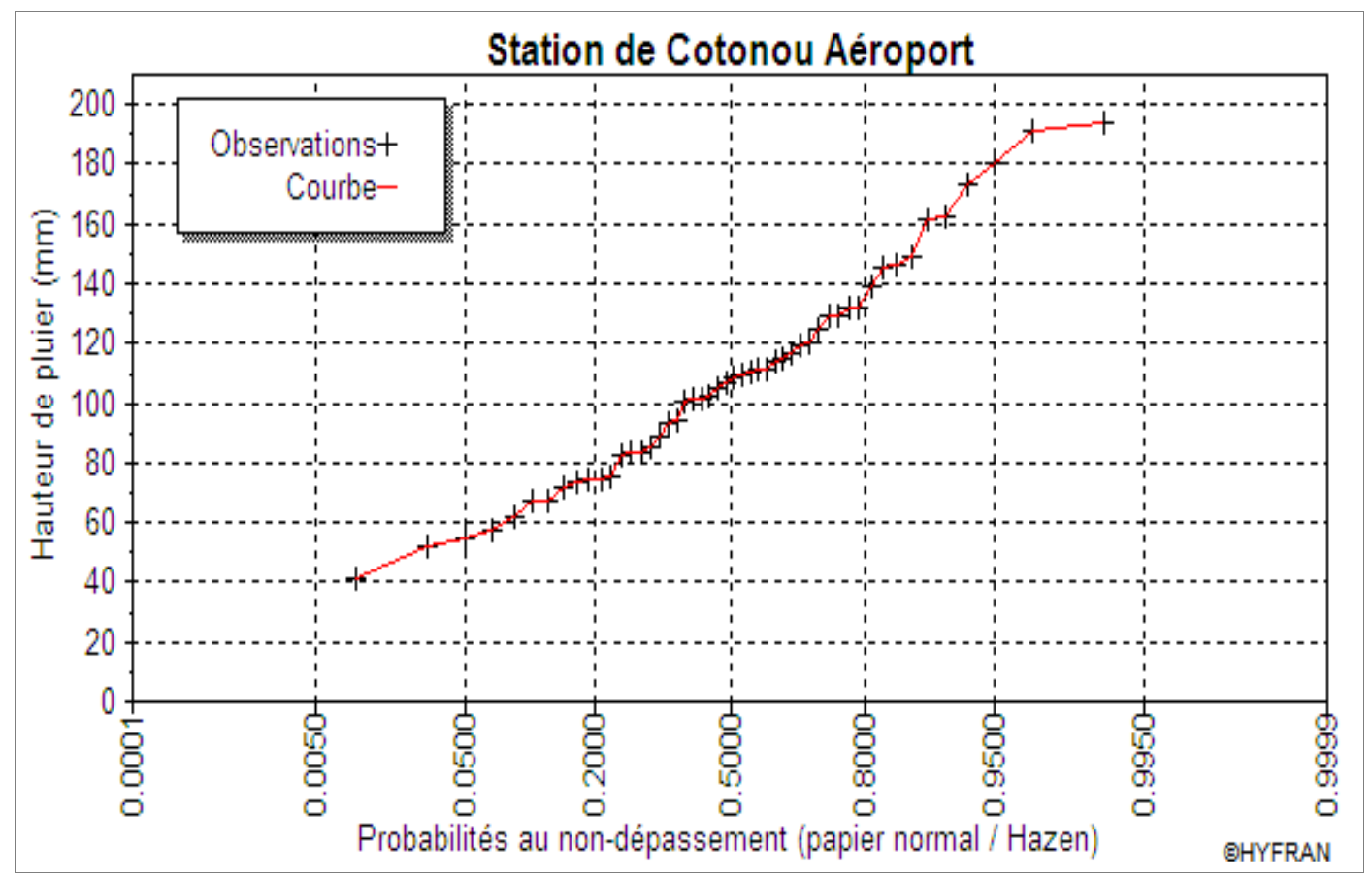

Figure 2a : Evolution de la probabilité empirique de la série des maxima annuels de pluies à Cotonou.

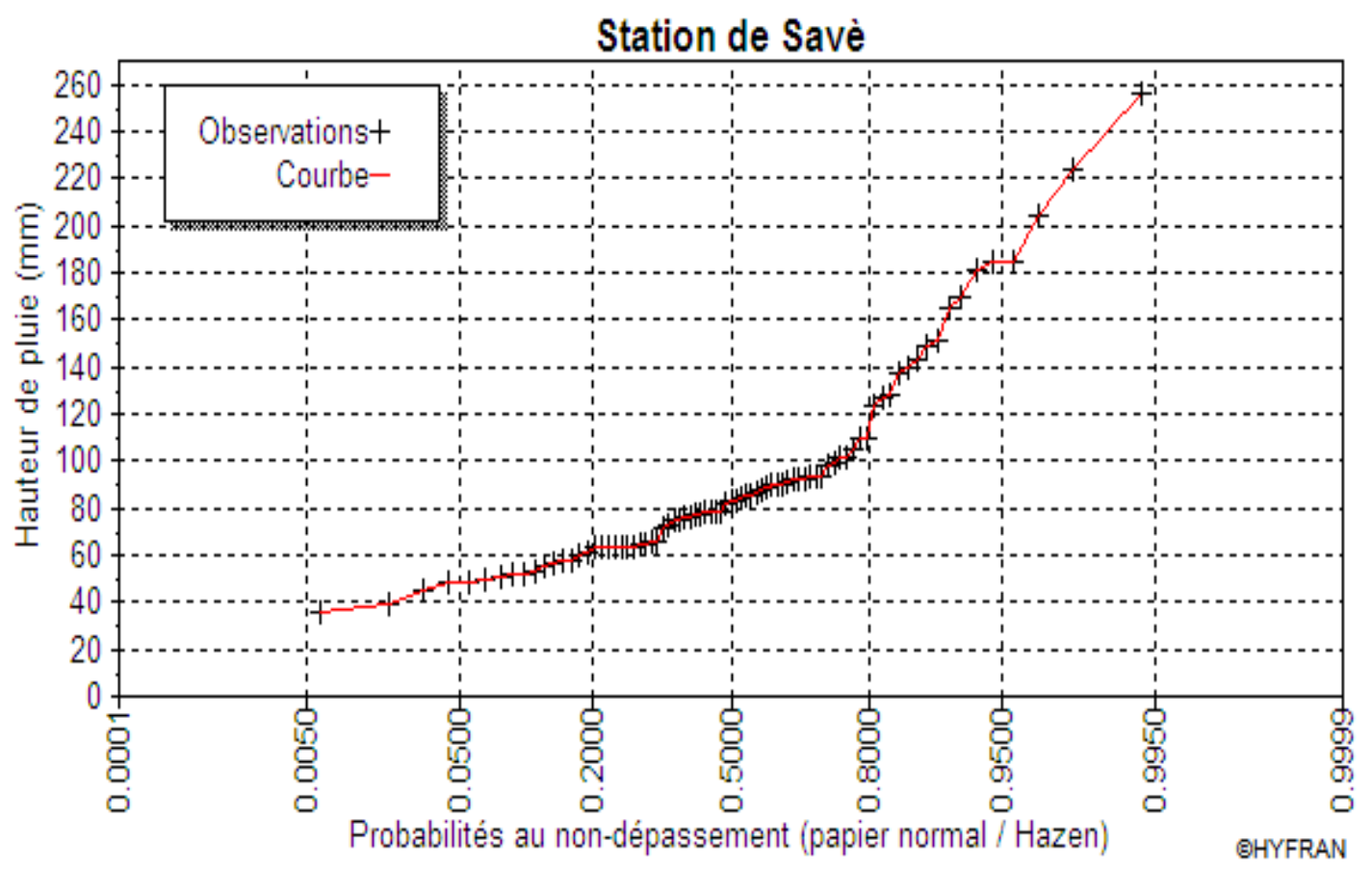

Figure 2b : Evolution de la probabilité empirique de la série des maxima annuels de pluies à Savè. 


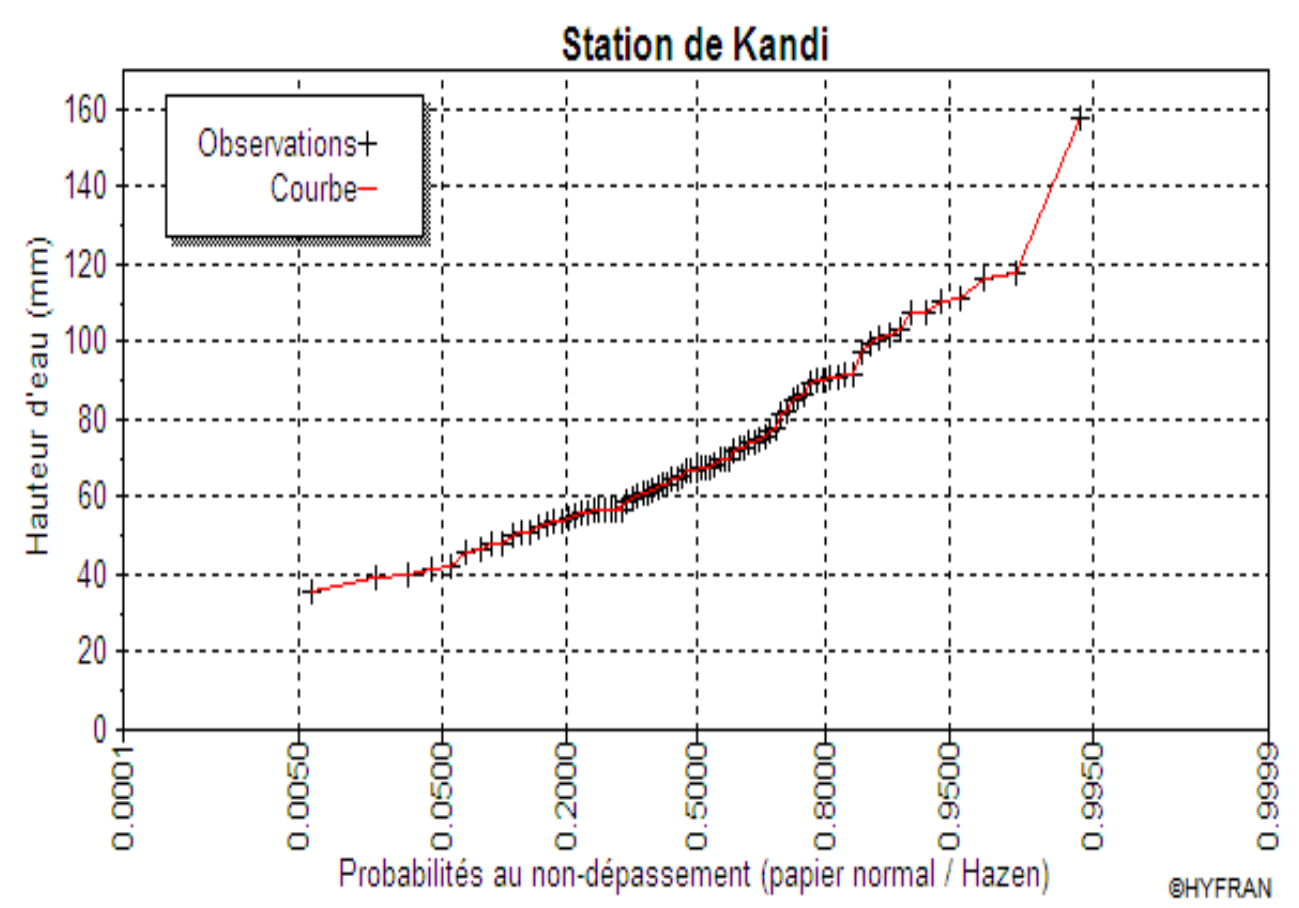

Figure 2c: Evolution de la probabilité empirique de la série des maxima annuels de pluies à Kandi.

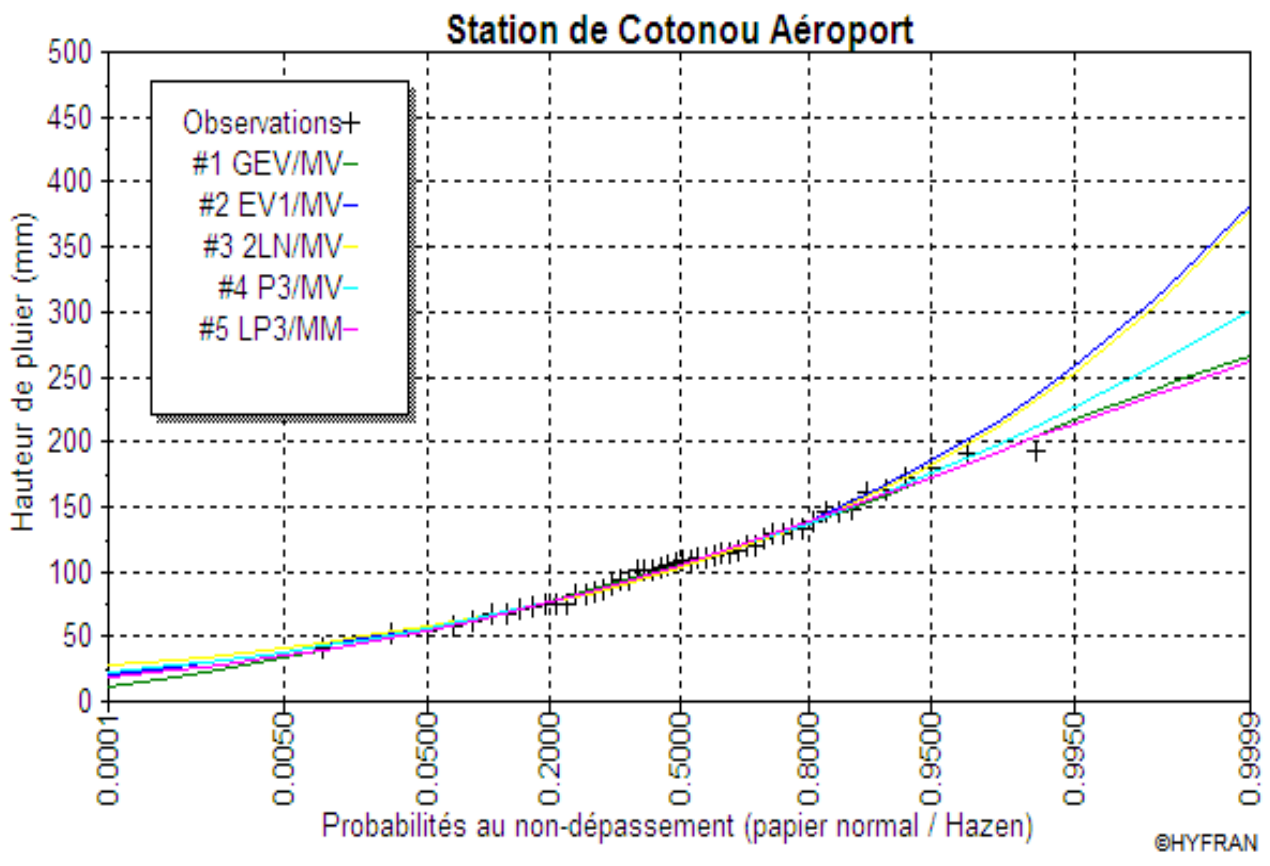

Figure 3 : Comparaison graphique des lois de probabilités appliquées à la série des données de Cotonou Aéroport. 


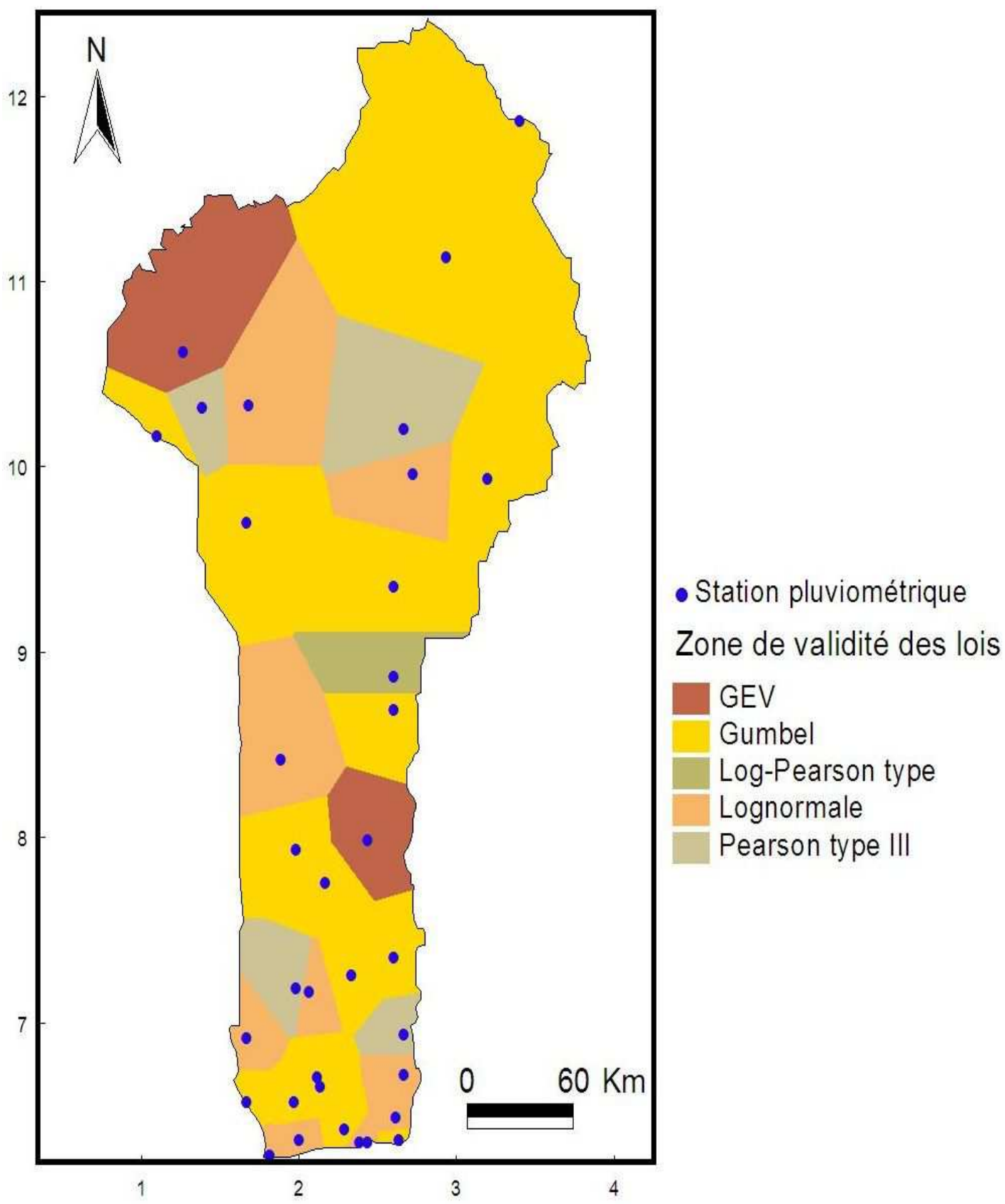

Figure 4 : Zone de validité des lois de probabilité. 

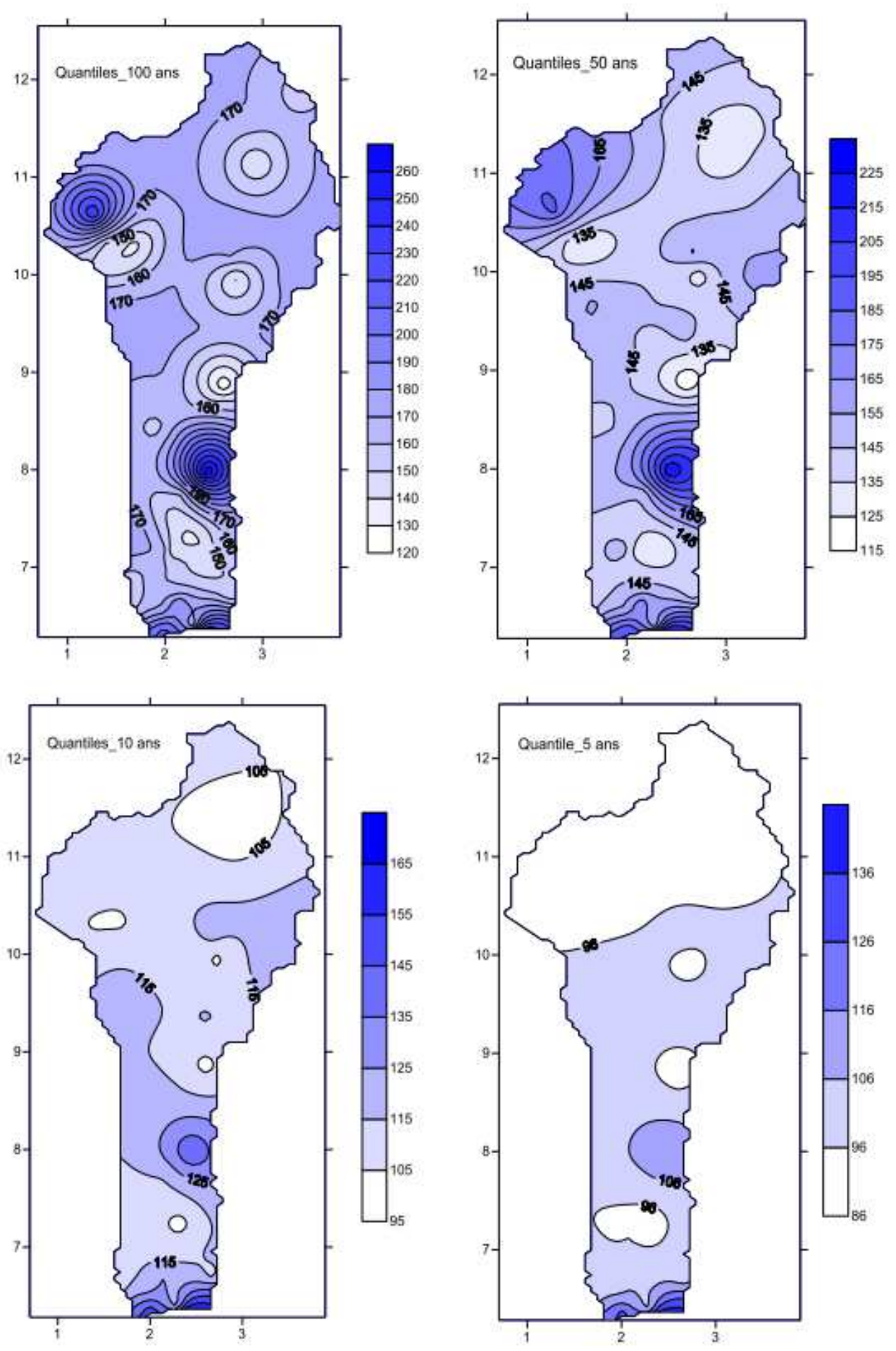

Figure 5 : Cartographie des quantiles de pluies journalières extrêmes par périodes de retour. 

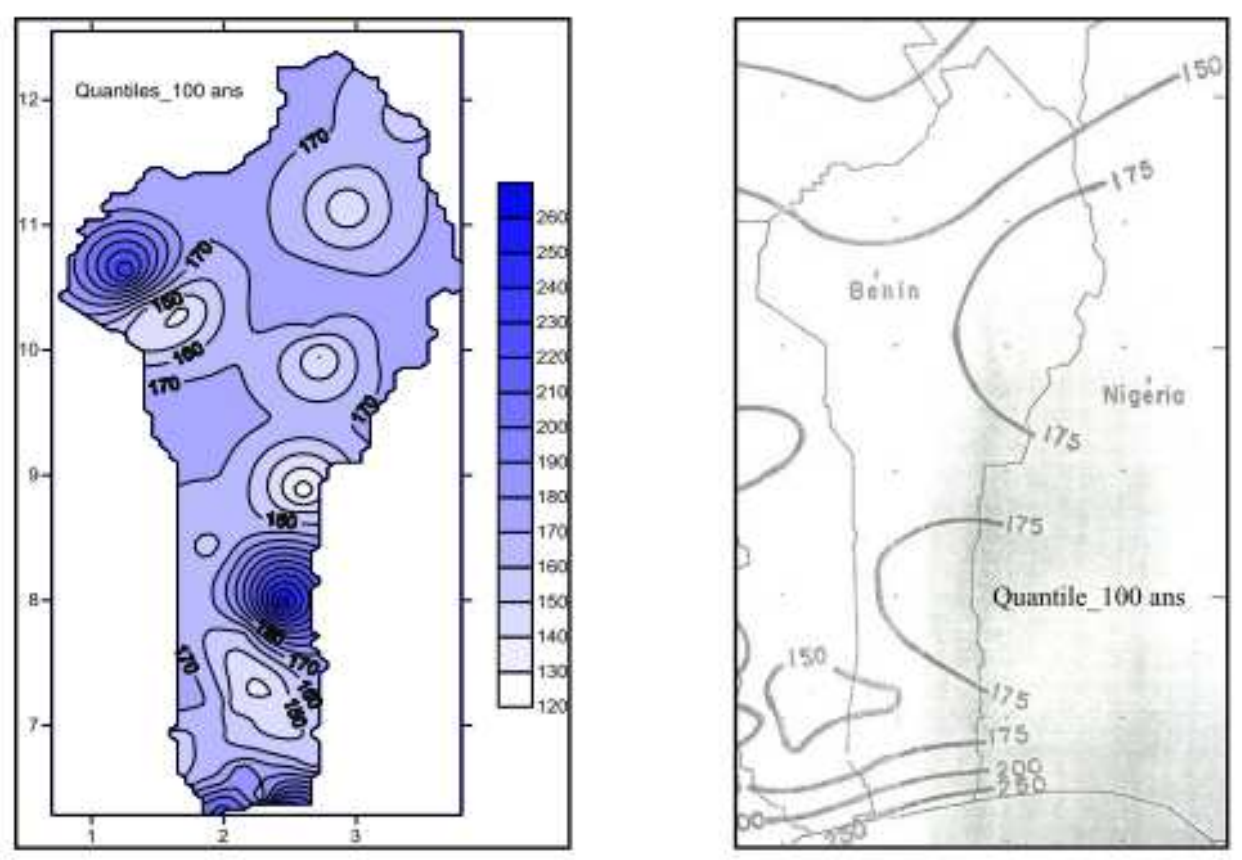

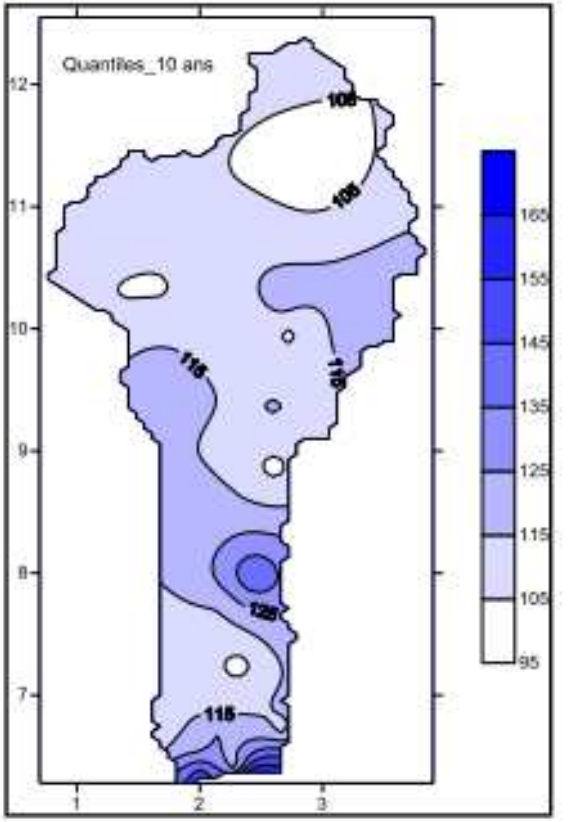

Etude Actuelle

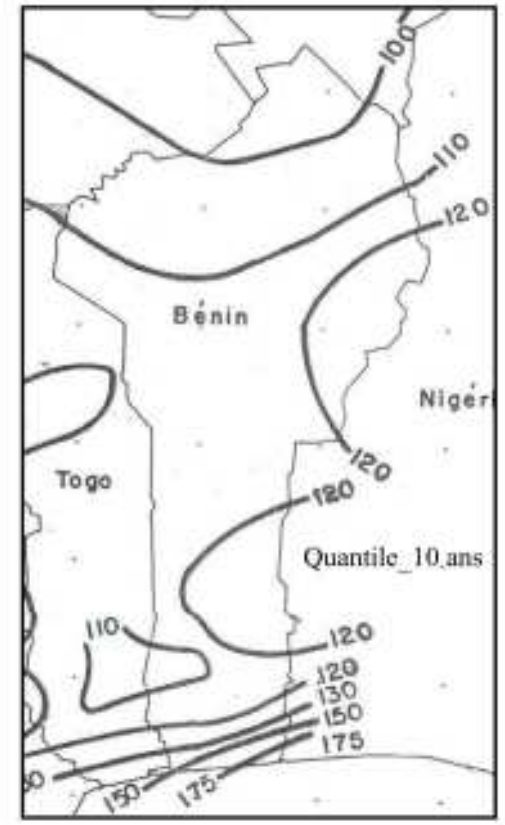

CIEH 1985

Figure 6 : Comparaison des isohyètes actuels et du CIEH (1985). 
Tableau 1 : Lois statistiques et leurs fonctions de densité de probabilité.

\begin{tabular}{llc}
\hline Loi & Fonction de densité de probabilité & Paramètres \\
\hline $\begin{array}{l}\text { Loi Généralisée des } \\
\text { Valeurs Extrêmes }\end{array}$ & $f(x)=\frac{1}{\alpha}\left[1-\frac{k}{\alpha}(x-u)\right]^{\frac{1}{k}-1} \exp \left\{-\left[1-\frac{k}{\alpha}(x-u)\right]^{\frac{1}{k}}\right\}$ & $\alpha, u, k$ \\
Gumbel & $f(x)=\frac{1}{\alpha} \exp \left[-\frac{x-u}{\alpha}-\exp \left(\frac{x-u}{\alpha}\right)\right]$ & $\alpha, u$ \\
Log Normal & $f(x)=\frac{1}{x \sigma \sqrt{2 \Pi}} \exp \left\{-\frac{(\ln x-\mu)^{2}}{2 \sigma^{2}}\right\}$ & $\sigma, \mu$ \\
Pearson Type III & $f(x)=\frac{\alpha^{\lambda}}{\Gamma(\lambda)}(x-m)^{\lambda-1} e^{-\alpha(x-m)}$ & $\alpha, \lambda, m$ \\
Log-Pearson type III & $f(x)=\frac{\alpha^{\lambda}}{\Gamma(\lambda)}(\ln x-m)^{\lambda-1} e^{-\alpha(x-m)}$ & $\alpha, \lambda, m$ \\
\hline
\end{tabular}

Tableau 2 : Critères de comparaison AIC et BIC des cinq lois de probabilité utilisées sur la série des données de la station de Cotonou Aéroport.

\begin{tabular}{|c|c|c|}
\hline Lois de probabilité & AIC & BIC \\
\hline Lognormale & 506.92 & 503.09 \\
\hline Gumbel & 507.07 & 503.25 \\
\hline Log-Pearson type III & 509.49 & 503.75 \\
\hline Pearson type III & 509.69 & 503.96 \\
\hline GEV & 509.77 & 504.03 \\
\hline
\end{tabular}

Tableau 2 : Quantiles calculés.

\begin{tabular}{cccccc}
\hline \multirow{2}{*}{ Stations } & Loi de probabilité & \multicolumn{5}{c}{ Quantiles $(\mathbf{m m})$} \\
\cline { 3 - 6 } & & P100 & P50 & P10 & P5 \\
\hline Savè & GEV & 270 & 226 & 145 & 117 \\
Tanguiéta & GEV & 240 & 188 & 109 & 86.7 \\
Cotonou Ville & Gumbel & 242 & 220 & 167 & 143 \\
Sèmè & Gumbel & 226 & 207 & 161 & 140 \\
Allada & Gumbel & 189 & 171 & 126 & 105 \\
Bopa & Gumbel & 173 & 157 & 121 & 104 \\
Niaouli & Gumbel & 173 & 158 & 122 & 106 \\
Savalou & Gumbel & 173 & 158 & 121 & 104 \\
Adjohoun & Gumbel & 172 & 157 & 120 & 103 \\
Nikki & Gumbel & 172 & 156 & 119 & 102 \\
Djougou & Gumbel & 171 & 156 & 120 & 104 \\
Parakou & Gumbel & 163 & 149 & 116 & 101 \\
Dassa-Zounmè & Gumbel & 161 & 148 & 117 & 103 \\
Kétou & Gumbel & 161 & 147 & 115 & 100 \\
Athiémé & Gumbel & 157 & 143 & 111 & 96.3 \\
\hline
\end{tabular}




\begin{tabular}{cccccc}
\hline Boukoumbé & Gumbel & 156 & 142 & 109 & 94.5 \\
Toui & Gumbel & 154 & 140 & 109 & 94.5 \\
Malanville & Gumbel & 152 & 138 & 105 & 89.6 \\
Kandi & Gumbel & 140 & 128 & 99.7 & 87 \\
Zangnannado & Gumbel & 139 & 128 & 102 & 89.9 \\
Cotonou Aérop & Lognormale & 232 & 211 & 161 & 138 \\
Ouidah Ville & Lognormale & 213 & 194 & 149 & 128 \\
Grand-popo & Lognormale & 210 & 189 & 142 & 120 \\
Porto-Novo & Lognormale & 206 & 189 & 148 & 129 \\
Aplahoué & Lognormale & 157 & 144 & 113 & 98.9 \\
Bantè & Lognormale & 157 & 145 & 115 & 100 \\
Sakété & Lognormale & 151 & 139 & 112 & 98.3 \\
Bohicon & Lognormale & 142 & 132 & 108 & 96 \\
Ina & Lognormale & 139 & 129 & 103 & 91.4 \\
Kouandé & Lognormale & 138 & 128 & 104 & 91.9 \\
Benbereke & Pearson type III & 171 & 156 & 119 & 102 \\
Abomey & Pearson type III & 166 & 150 & 111 & 94.2 \\
Pobè & Pearson type III & 152 & 143 & 118 & 105 \\
Natitingou & Pearson type III & 149 & 135 & 104 & 88.8 \\
Tchaourou & Log-Pearson type III & 122 & 117 & 102 & 92.7 \\
\hline
\end{tabular}

\section{Conclusion}

La présente étude fait l'analyse fréquentielle et propose une nouvelle cartographie des maxima annuels des pluies journalières au Bénin. La taille de toutes nos séries est supérieure à 30 ans. Malgré la dominance des lois Gumbel et Lognormale, les autres lois admettent également des zones de validité. On retient que l'usage d'une ou au plus deux lois, comme dans les anciennes études, pourrait ne pas permettre, une bonne estimation des quantiles des pluies journalières au Bénin. La cartographie des quantiles a révélé la caducité des isohyètes proposés par le CIEH et très souvent utilisés dans le dimensionnement des ouvrages hydrauliques. Il apparait donc judicieux de considérer les nouvelles propositions pour garantir une sécurité des ouvrages dans le contexte actuel de changement climatique. Dans le souci d'apprécier l'impact du changement climatique sur la variabilité des extrêmes, nous envisageons reprendre l'étude en considérant les données par décennie afin d'apprécier des différences significatives d'une décennie à une autre.

\section{REMERCIEMENTS}

Nos gratitudes vont à l'endroit de la Chaire Internationale en Physique Mathématique et Applications de l'Université d'Abomey-Calavi, de $\mathrm{M}^{\text {me }}$ HOUGUE Rita et $\mathrm{Mr}$ KOUHOUNDJI Naboua pour leur assistance technique puis de l'Agence pour la Sécurité de la Navigation Aérienne en Afrique et au Madagascar (ASECNA) pour avoir mis à notre disposition les données de pluie.

\section{REFERENCES}

Aka A, Servat E, Paturel JE, Kouamé B, Lubes H, Masson JM. 1996. Analysis of the temporal variability of runoff in Ivory Coast: statistical approach and phenomena characterization. Hydrological Sciences Journal, 41(6): 959-970.

Benkhaled A. 2007. Distributions statistiques des pluies maximales annuelles dans la région du Cheliff : comparaison des techniques et des résultats. Courrier $d u$ Savoir, 8: 83 - 91.

Bouvier CH, Ramon D, Luc N, Hélène N. 2005. Approche régionale pour l'estimation des distributions ponctuelles 
des pluies journalières dans le Languedoc-Roussillon. Hydrological Sciences Journal, 50(1): 17-29.

CIEH.1985. Etude des pluies journalières de fréquence rare dans les pays membres du CIEH. Rapport de synthèse, $58 \mathrm{p}$.

Darricau H. 1980. Les pluies journalières maximales annuelles moyennes en HauteVolta. Ecole Normale Supérieure des Mines Paris, Centre d'Informations Géographiques.

Goula BT, Brou K, Brou T, Savane I, Vamoryba F, Bernard S. 2007. Estimation des pluies exceptionnelles journalières en zone tropicale: cas de la Côte d'Ivoire par comparaison des lois Lognormale et de Gumbel. Hydrological Sciences-Journaldes Sciences Hydrologiques, 52(1): 4967.

Goula BTA, Soro G E, Dao A, Kouassi FW, Srohourou B. 2010. Frequency analysis and new cartography of extremes daily rainfall events in Côte d'Ivoire. Journal of Applied Sciences, 10: 1684 - 1694.

Hache M, Perreault L, Remillard L, Bobee B. 1999. Une approche pour la sélection des distributions statistiques: application au bassin hydrographique du Saguenay Lac St Jean. Revue Canadienne de Génie Civil. 26(2): 216-225.

Koutsoyiannis D, Baloutsos G. 2000. Analysis of a long record of annual maximum rainfall in Athens, Greece and design rainfall inferences. Natural Hazards (Dordrecht), 22(1): 29-48.

Koutsoyiannis D. 2004a. Statistics of estimation of extreme rainfall: I. Theoretical of investigation. Journal des Sciences Hydrologiques, 49(4) : 575-590.

Koutsoyiannis D. 2004b. Statistics of estimation of extreme rainfall: II. Theoretical of investigation. Empirical investigation of long rainfall records. Journal des Sciences Hydrologiques, 49(4) : 591-610.

Koutsoyiannis D. 2005. Incertitude, entropie, effet d'échelle et propriétés stochastiques hydrologiques.

Propriétés distributionnelles marginales des processus hydrologiques et échelle d'état. Journal des Sciences Hydrologiques, 50(3) : 405-426.

LeBartier E, Mary-Huard T. 2004. Le critère BIC: fondements théoriques et interprétation. Rapport de recherche, Institut National de la Recherche en Information et en Automatique (France), $16 \mathrm{p}$.

Panigrahi B, Sudhindra NP. 2002. Prediction of hydrological events for planning rain fed rice. Hydrological Sciences Journal, 47(3): 435-448.

Siegel S. 1956. Non-Parametric Statistics for the Behavioral Sciences. McGraw-Hill: New York.

Soro GE, Goula Bi TA, Kouassi FW, Koffi K, Kamagate B, Doumouya I, Savane I, Srohorou B. 2008. Courbes Intensité Durée Fréquence des précipitations En climat Tropical Humide: Cas de la Région d'Abidjan (Côte D'Ivoire). European Journal of Scientific Research, 21(3): 394-405.

Soro GE. 2010. Modélisation statistique des pluies extrêmes en Côte d'Ivoire. Thèse unique de doctorat, Université Nangui Abrogoua, Côte d'Ivoire, p.101.

Van de Vyver H, Demarée GR. 2010. Construction of Intensity-DurationFrequency (IDF) curves for precipitation at Lubumbashi, Congo, under the hypothesis of inadequate data. Hydrological Sciences Journal, 55(4): $555-564$

Zahar Y, Laborde JP. 2007. Modélisation statistique et synthèse cartographique des pluies journalières extrêmes de Tunisie. Rev. Sci. Eau, 20: 409-424. 